University of Nebraska - Lincoln

DigitalCommons@University of Nebraska - Lincoln

November 1990

\title{
Kinetic-energy density functional for a special shape-invariant potential of a one-dimensional two-level system
}

Jiushu Shao

Institut für Organische Chemie, Biochemie und Isotopenforschung, Universität Stuttgart

John J. Stezowski

University of Nebraska - Lincoln, jjs@unlserve.unl.edu

Follow this and additional works at: https://digitalcommons.unl.edu/chemistrystezowski

Part of the Chemistry Commons

Shao, Jiushu and Stezowski, John J., "Kinetic-energy density functional for a special shape-invariant potential of a one-dimensional two-level system " (1990). John J. Stezowski Publications. 2.

https://digitalcommons.unl.edu/chemistrystezowski/2

This Article is brought to you for free and open access by the Published Research - Department of Chemistry at DigitalCommons@University of Nebraska - Lincoln. It has been accepted for inclusion in John J. Stezowski Publications by an authorized administrator of DigitalCommons@University of Nebraska - Lincoln. 


\title{
Rapid Communications
}

The Rapid Communications section is intended for the accelerated publication of important new results. Since manuscripts submitted to this section are given priority treatment both in the editorial office and in production, authors should explain in their submittal letter why the work justifies this special handling. A Rapid Communication should be no longer than $31 / 2$ printed pages and must be accompanied by an abstract. Page proofs are sent to authors.

\section{Kinetic-energy density functional for a special shape-invariant potential of a one-dimensional two-level system}

\author{
Jiushu Shao and John J. Stezowski \\ Institut für Organiche Chemie, Biochemie und Isotopenforschung, Universität Stuttgart, Pfaffenwaldring 55, \\ 7000 Stuttgart 80, Federal Republic of Germany
}

(Received 17 July 1990)

\begin{abstract}
By introducing the concept of supersymmetry to quantum mechanics, several authors have shown that exactly soluable potentials including all those well known are easily constructed. Is the kinetic-energy density functional corresponding to these potentials a simple form? We show that the answer is no, even to the simplest one, the harmonic potential, if one builds the kineticenergy density functional from the reduced density matrix.
\end{abstract}

Since the very beginning of modern density-functional theory ${ }^{1}$ (DFT), many efforts have been made to search for the energy density functional, ${ }^{2}$ especially the kineticenergy density functional ${ }^{3}$ (KEDF). But no one has found the exact functional except for the one-particle system that is trivial in DFT. In fact, the approximate formulation, e.g., the functional in the approximation of gradient expansion, is frequently used in the application of DFT to atoms and molecules. ${ }^{4}$

It has been known recently that with the help of supersymmetry and the concept of shape invariance, one can easily treat the quantum systems of all well-known exactly solvable potentials. ${ }^{5}$ It seems that such systems would also be easily dealt with in DFT. Now the question arises: can one find the KEDF to the two-particle system? Even for the system having a simply shape-invariant potential, we shall show in the following that the answer is negative.

Consider two noninteracting spinless fermions moving in the external potential $V_{-}$. Assume $V_{-}$is shape invariant and its supersymmetric partner $V_{+}$satisfies

$$
V_{+}=V_{-}+R
$$

where $R$ is a constant.

For this system, then, if its lowest single-particle wave function is $\psi_{0}(x)$, its next-to-the-lowest single-particle wave function is

$$
\psi_{1}(x)=\frac{2}{\sqrt{\varepsilon}} \psi_{0}^{\prime}(x),
$$

where $\varepsilon$ is the energy difference between the higher and the lower orbit.

For a given density $\rho(x)$, we have

$$
\psi_{0}^{2}+\psi_{1}^{2}=\rho(x)
$$

or

$$
\left(\frac{2}{\sqrt{\varepsilon}} \psi_{0}^{\prime}\right)^{2}+\psi_{0}^{2}=\rho(x)
$$

Equation (4) may be transformed to the Abel equation that is not exactly solvable, and we will not discuss the details here.

Following Aryasetiawan and Stott, ${ }^{6}$ we set the transformation

$$
\begin{aligned}
& \psi_{0}=\sqrt{\rho} \cos \theta(x), \\
& \psi_{1}=\sqrt{\rho} \sin \theta(x),
\end{aligned}
$$

and obtain

$$
\theta^{\prime \prime}+\frac{\rho^{\prime}}{\rho} \theta^{\prime}+\varepsilon \sin (2 \theta)=0
$$

and the kinetic-energy density may be derived as ${ }^{7}$

$$
t=-\frac{1}{4} \rho^{\prime \prime}+\frac{1}{8}\left(\frac{\rho^{\prime}}{\rho}\right)^{2}+\frac{1}{2} \rho\left(\theta^{\prime}\right)^{2}
$$

From Eqs. (4) and (5), one gets

$$
\begin{aligned}
& \theta^{\prime}=\frac{1}{2} \frac{\rho^{\prime}}{\rho} \cot \theta-\frac{1}{2} \sqrt{\varepsilon} \\
& \theta^{\prime \prime}=\frac{1}{2}\left(\frac{\rho^{\prime}}{\rho}\right)^{\prime} \cot \theta-\frac{1}{2} \frac{\rho^{\prime}}{\rho} \csc ^{2} \theta\left(\frac{1}{2} \frac{\rho^{\prime}}{\rho} \cot \theta-\frac{1}{2} \sqrt{\varepsilon}\right) .
\end{aligned}
$$

Substituting Eqs. (8) and (9) into Eq. (6), we have 
$\frac{1}{2}\left(\frac{\rho^{\prime}}{\rho}\right)^{\prime} \cot \theta-\frac{1}{2} \frac{\rho^{\prime}}{\rho} \csc ^{2} \theta\left(\frac{1}{2} \frac{\rho^{\prime}}{\rho} \cot \theta-\frac{1}{2} \sqrt{\varepsilon}\right)+\frac{\rho^{\prime}}{\rho}\left(\frac{1}{2} \frac{\rho^{\prime}}{\rho} \cot \theta-\frac{1}{2} \sqrt{\varepsilon}\right)+\varepsilon \sin (2 \theta)=0$

Equation (10) is an algebraic equation of $\theta$. To simplify it, let $\tan \theta=x$ and $\rho^{\prime} / \rho=\alpha$, and after trivial arithmetic manipulation Eq. (9) reads

$x^{5}+\frac{1}{\alpha \sqrt{\varepsilon}}\left(\alpha^{2}+2 \alpha^{\prime}+8 \varepsilon\right) x^{4}+\frac{2}{\sqrt{\varepsilon}} x^{2}-x-\frac{1}{\sqrt{\varepsilon}} \alpha=0$.
If we got $\theta$, we would get the KEDF through Eq. (7). However, Eq. (11) is not solvable analytically, thus the exact $\theta$ is inaccessible. Therefore, we have shown that one cannot expect that quantum systems with solvable potentials are also "solvable" in DFT if one sets up the KEDF from the reduced density matrix, except the case in which $\rho^{\prime} / \rho$ is a constant. ${ }^{8}$
'P. Hohenberg and W. Kohn, Phys. Rev. B 136, 864 (1964).

${ }^{2}$ Theory of the Inhomogeneous Electron Gas, edited by $\mathrm{S}$. Lundqvist and N. H. March (Plenum, New York, 1983).

${ }^{3}$ C. H. Hodges, Can. J. Phys. 51, 4128 (1973).

${ }^{4}$ W-P. Wang, R. G. Parr, D. R. Murphy, and G. A. Henderson, Chem. Phys. Lett. 43, 409 (1976); N. L. Allan and D. L. Cooper, J. Chem. Phys. 84, 5594 (1986).

${ }^{5}$ R. Dutt, A. Khare, and U. P. Sukhatme, Am. J. Phys. 56, 163 (1988).

${ }^{6}$ F. Aryasetiawan and M. J. Stott, Phys. Rev. B 34, 4401 (1986).
${ }^{7}$ N. H. March and R. F. Nalewajski, Phys. Rev. A 35, 525 (1987).

${ }^{8}$ When $\theta^{\prime} / \rho$ is a constant, one can get the exact KEDF and only then the approximation of the gradient expansion becomes exact [cf. J. Shao, J. J. Stezowski, and R. Baltin (unpublished)]. It is easy to show that $V$ - is the harmonic potential. Kozlowski and March [Phys. Rev. A 39, 4270 (1989)] claim to have obtained the explicit form the KEDF for the $n$-level system with harmonic potential, but their work is based on the local-density approximation, although the authors seem to neglect this point in their paper. 